\title{
Y-chromosomal DNA haplotype differences in control and infertile Italian subpopulations
}

\author{
Carlo Previderé ${ }^{1 *}$, Liborio Stuppia ${ }^{2,3}$, Valentina Gatta ${ }^{2}$, Paolo Fattorini ${ }^{4}$, \\ Giandomenico Palka ${ }^{2}$ and Chris Tyler-Smith ${ }^{1}$ \\ ${ }^{1}$ CRC Chromosome Molecular Biology Group, Department of Biochemistry, University of Oxford, UK \\ ${ }^{2}$ Dipartimento di Scienze Biomediche, Sezione di Genetica Medica, Università degli studi 'G D'Annunzio', Chieti \\ ${ }^{3}$ Istituto di Citomorfologia Umana Normale e Patologica CNR, Chieti \\ ${ }^{4}$ Istituto di Medicina Legale e delle Assicurazioni, Trieste, Italy
}

\begin{abstract}
Y-chromosomal DNA haplotypes were determined in $\mathbf{7 4}$ infertile and 216 control Italian males using eight biallelic markers. A significant difference in haplotype frequency was found, but could be explained by the geographical origins of the samples. The Y chromosome is thus a sensitive marker for population substructuring and may be useful for determining whether two population samples come from a single population, for example in association studies.
\end{abstract}

Keywords: Y-chromosomal DNA haplotype; biallelic marker; SNP; male infertility; population substructure

\section{Introduction}

Infertility is estimated to affect $2-7 \%^{1}$ or $10 \%^{2}$ of males. In over $10 \%$ of infertile patients, representing almost $1 \%$ of the population, there is oligozoospermia or azoospermia with no obvious cause, described as 'idiopathic'. The Y chromosome carries several genes required for male fertility. De novo deletions of the $A Z F a, A Z F b$ or $A Z F c$ loci on Yq together account for a proportion of idiopathic infertility cases, estimated at $3 \%(12 / 370)$ in one survey and $18 \%(6 / 33)$ in another, ${ }^{4}$ but the cause of infertility in most of the remaining cases is unknown. It is possible that other mutations or

Correspondence: Chris Tyler-Smith, CRC Chromosome Molecular Biology Group, Department of Biochemistry, University of Oxford, South Parks Road, Oxford OX1 3QU, UK. Tel: + 441865 275222; Fax: + 441865 275259; E-mail: chris@bioch.ox.ac.uk.

*Present address: Dipartimento di Medicina Legale e Sanita Pubblica, Via Forlanini 12, 27100 Pavia, Italy.

Received 17 February 1999; revised 9 April 1999; accepted 13 April 1999 genes on the $\mathrm{Y}$ chromosome are involved. Although mutations on the Y leading to infertility would not be transmitted to the next generation, a predisposition to such a mutation could be transmitted. Such a predisposition might be recognised directly through an understanding of its molecular mechanism, or indirectly through its occurrence on a particular haplotypic background. We have previously identified an association between one rare class of infertile males, $P R K X /$ $P R K Y$ translocation $\mathrm{XX}$ males, and a particular $\mathrm{Y}$ haplotype. ${ }^{5}$ In this case, it was suggested that an inversion polymorphism on Yp which includes the PRKY gene allows efficient alignment and ectopic recombination with $P R K X$ in one orientation but not in the other. We therefore set out to look for more general associations between Y haplotypes and infertility in a set of Italian patients and controls. Such associations would be important because they would indicate that novel Y defects or genes contribute to infertility, and also because they might reveal differential selective forces acting on Y haplotypes. 


\section{Materials and Methods}

\section{DNA Samples}

The infertile males were 74 patients with idiopathic infertility presenting at the University of Chieti. All patients were carefully examined in order to rule out any known cause of infertility and classified according to WHO criteria. ${ }^{6}$ Fifteen patients were azoospermic, 52 were oligozoospermic and the remaining seven had abnormal sperm morphology and/or motility. Deletions of Yq loci within interval 6 including the $D A Z$ gene cluster ${ }^{7}$ were detected in two of the 55 individuals tested. 65 of the patients (88\%) were from central Italy. Unrelated normal males used as controls were a set with proven fertility from central Italy $(n=59)$, and random males ( $>90 \%$ expected to be fertile) from northwestern $(n=99)$ and northeastern $(n=58)$ Italy.

\section{Markers}

The biallelic Y markers used were the presence or absence of the Alu insertion $\mathrm{YAP}^{8}$ and seven base substitution polymorphisms: $S R Y-8299\left(\mathrm{G} \rightarrow \mathrm{A} ; 4064^{9}\right)$, sY81 $(\mathrm{A} \rightarrow \mathrm{G}),{ }^{10} \mathrm{M} 9$ $(\mathrm{C} \rightarrow \mathrm{G}){ }^{11}$ 92R7 $(\mathrm{C} \rightarrow \mathrm{T})^{12}$ in PCR format, SRY-1532 $(\mathrm{A} \rightarrow \mathrm{G} \rightarrow \mathrm{A}){ }^{13} S R Y-2627\left(\mathrm{C} \rightarrow \mathrm{T}\right.$; also called $\left.S R Y-2628^{14}\right)$ and LLY22g/HindIII $(\mathrm{C} \rightarrow \mathrm{A}){ }^{15}$ All markers are located in the Y-specific portion of the chromosome. Those designated $S R Y-\mathrm{X}$ are located ' $\mathrm{X}$ ' bp upstream of the $S R Y$ gene on $\mathrm{Yp}$; 92R7 is also on Yp. ${ }^{16}$ The other markers are located in Yq in intervals $5 \mathrm{~A}$ (sY81), 5O (YAP, M9) ${ }^{17}$ or 17 (LLY22g/ HindIII). ${ }^{16}$

\section{Results}

Y-chromosomal DNA haplotypes were determined for 290 Italian males. In a worldwide survey, the eight markers used could together distinguish ten different haplotypes, referred to as 'haplogroups', $\left({ }^{18}\right.$ and results not shown). A parsimony network illustrating the relationships between these haplogroups is shown in Figure 1a. Only six haplogroups (numbered in Figure 1a) were found in Italy. The two commonest were haplogroups 1 and 2, which differ at the loci M9 and 92R7. Overall, 57\% (165/290) of the chromosomes tested carried the M9 C allele, while the remaining $43 \%$ $(125 / 290)$ carried the $\mathrm{G}$ allele; with 92R7, 62\% (180/290) carried the C allele, and the remaining 38\% (110/290) carried the T allele (Table 1, Figure 1b). These frequencies are not, of course, independent. For this reason, we consider only the $92 \mathrm{R} 7$ results, but the same conclusions were reached using M9. There were statistically significant differences between the frequencies of the 92R7 alleles in the infertile males and three normal control populations grouped together; $73 \%$ (54/74) of the infertile males carried the $92 \mathrm{R} 7 \mathrm{C}$, whilst only $58 \%$ $(126 / 216)$ of the pooled controls carried the C. This difference is significant at the $5 \%$ level $\left(P=0.027, \chi^{2}\right.$ or G-test). a

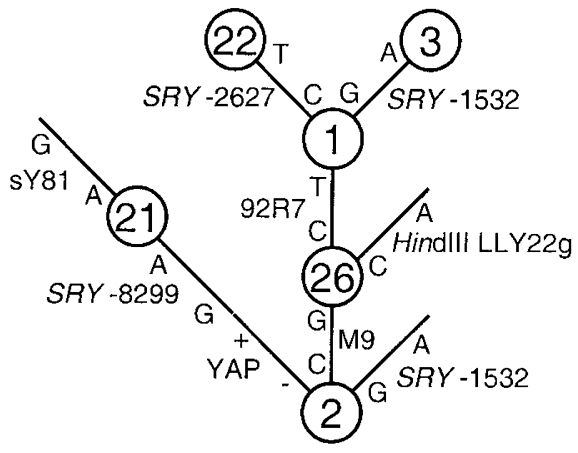

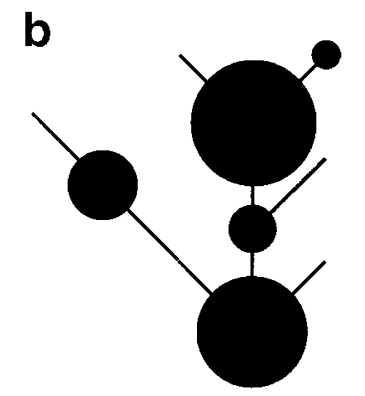

NW Italy $(n=99)$

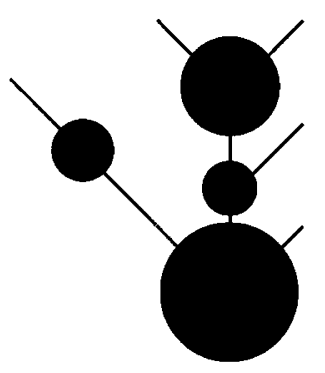

central Italy $(n=59)$

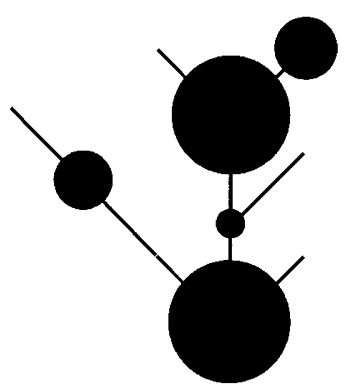

NE Italy $(n=58)$

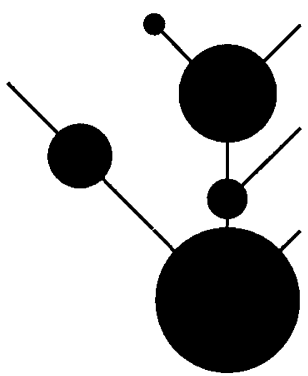

infertile males $(n=74)$
Figure 1 (a) Markers and the haplogroups they define. In the phylogenetic tree, the markers used are indicated along the lines, together with the two bases found, or the absence (-) or presence (+) of the retroposon insertion YAP. The haplogroups present in the Italian samples are indicated by numbers inside circles. (b) Relative frequencies of haplogroups in the Italian population samples. Frequency is proportional to the area of the circle.

Nevertheless, further examination of the data suggests that the frequency differences are due to geographical variation rather than a link between the $\mathrm{C}$ allele chromosomes and infertility. Most (65/74, 88\%) of the infertile males came from central Italy. Among these, 48 (74\%) carried the 92R7 C allele; among the control males from central Italy, 73\% (43/59) carried the $\mathrm{C}$. The difference is not statistically significant. 
Table 1 Y-chromosomal DNA haplotypes of infertile and control males

\begin{tabular}{lccccccc}
\hline Sample & $n$ & \multicolumn{6}{c}{ Haplogroup } \\
& & 1 & 2 & 3 & 21 & 22 & 26 \\
\hline Infertile & 74 & 19 & 43 & - & 8 & 1 & 3 \\
& & & & & & & \\
Controls & & & & & & & \\
NW Italy & 99 & 44 & 34 & 2 & 13 & - & 6 \\
NE Italy & 58 & 22 & 24 & 6 & 5 & - & 1 \\
C Italy & 59 & 16 & 32 & - & 6 & - & 5 \\
\hline
\end{tabular}

Differences between the central Italian controls and either of the northern control populations were significant $(P=0.014$, northwest; $P=0.022$, northeast $)$. The sample of infertile males from outside central Italy $(n=9)$ was too small for separate statistical analysis.

\section{Discussion}

The Y chromosome is known to show relatively high levels of geographical specificity compared with autosomal or mitochondrial DNA sequences, probably as a result of its small effective population size and/or a lower geographical mobility of males. ${ }^{11,19,20}$ Nevertheless, it is surprising to find such striking differences within the Italian population. A possible explanation could be that there are higher levels of inbreeding and, consequently, drift within the central Italian population sampled. Overall, these results suggest that the $\mathrm{Y}$ chromosome is the most sensitive locus in the genome to genetic differentiation, and could provide the best internal control to test whether two population samples, such as affected and control individuals in an association study, are well matched. Indeed, a comparison of Y haplotypes could be a useful initial step in any association study. A consequence of these findings is that associations between $\mathrm{Y}$ haplotypes and phenotypes will be particularly difficult to distinguish from population differences. Despite the lack of a significant difference between our samples of infertile and control males from central Italy, it remains possible that some Y haplotypes do predispose to infertility. However, a different strategy, such as comparing $\mathrm{Y}$ haplogroups among different molecular classes of $\mathrm{Y}$ deletion, may be required to reveal such predispositions.

\section{Acknowledgements}

We thank the donors of the DNA samples for making this study possible. CTS was supported by the CRC.

\section{References}

1 Elliot DJ, Cooke HJ: The molecular genetics of male infertility. BioEssays 1997; 19: 801-809.

2 Okabe M, Ikawa M, Ashkenas J: Male infertility and the genetics of spermatogenesis. Am J Hum Genet 1998; 62: 1274-1281.

3 Vogt PH, Edelmann A, Kirsch $\mathrm{S}$ et al: Human $\mathrm{Y}$ chromosome azoospermia factors (AZF) mapped to different subregions in Yq11. Hum Mol Genet 1996; 5: 933-943.

4 Stuppia L, Mastroprimiano G, Calabrese G, Peila R, Tenaglia R, Palka G: Microdeletions in interval 6 of the Y chromosome detected by STS-PCR in 6 of 33 patients with idiopathic oligo- or azoospermia. Cytogenet Cell Genet 1996; 72: 155-158.

5 Jobling MA, Williams G, Schiebel $\mathrm{K}$ et al: A selective difference between human Y-chromosomal DNA haplotypes. Curr Biol 1998; 8: 1391-1394.

6 World Health Organization: WHO Laboratory Manual for the Examination of Human Semen and Cervical Mucus Interaction, 3rd edn. Cambridge University Press: Cambridge, 1992.

7 Reijo R, Lee T-Y, Salo P et al: Diverse spermatogenic defects in humans caused by $\mathrm{Y}$ chromosome deletions encompassing a novel RNA-binding protein gene. Nat Genet 1995; 10: 383-393.

8 Hammer MF, Horai S: Y chromosomal DNA variation and the peopling of Japan. Am J Hum Genet 1995; 56: 951-962.

9 Whitfield LS, Sulston JE, Goodfellow PN: Sequence variation of the human Y chromosome. Nature 1995; 378: 379-380.

10 Seielstad MT, Hebert JM, Lin AA et al: Construction of human Y-chromosomal haplotypes using a new polymorphic A to G transition. Hum Mol Genet 1994; 3: 2159-2161.

11 Underhill PA, Jin L, Lin AA et al: Detection of numerous $\mathrm{Y}$ chromosome biallelic polymorphisms by denaturing high-performance liquid chromatography. Genome Res 1997; 7: 996-1005.

12 Mathias N, Bayes M, Tyler-Smith C: Highly informative compound haplotypes for the human Y chromosome. Hum Mol Genet 1994; 3: 115-123.

13 Kwok C, Tyler-Smith C, Mendonca BB et al: Mutation analysis of the $2 \mathrm{~kb} 5^{\prime}$ to SRY in XY females and XY intersex subjects. $J$ Med Genet 1996; 33: 465-468.

14 Veitia R, Ion A, Barbaux S et al: Mutations and sequence variants in the testis-determining region of the $\mathrm{Y}$ chromosome in individuals with a $46, \mathrm{XY}$ female phenotype. Hum Genet 1997; 99: 648-652.

15 Zerjal T, Dashnyam B, Pandya A et al: Genetic relationships of Asians and northern Europeans, revealed by Y-chromosomal DNA analysis. Am J Hum Genet 1997; 60: 1174-1183.

16 Tyler-Smith C, Oakey RJ, Larin Z et al: Localization of DNA sequences required for human centromere function through an analysis of rearranged Y chromosomes. Nat Genet 1993; 5: 368-375.

17 Vollrath D, Foote S, Hilton A et al: The human Y chromosome: a 43-interval map based on naturally occurring deletions. Science 1992; 258: 52-59. 
18 Vogt PH, Affara N, Davey P et al: Report of the third international workshop on Y chromosome mapping 1997. Cytogenet Cell Genet 1997; 79: 1-20.

19 Jobling MA, Tyler-Smith C: Fathers and sons: the Y chromosome and human evolution. Trends Genet 1995; 11: 449-456.
20 Seielstad MT, Minch E, Cavalli-Sforza LL: Genetic evidence for a higher female migration rate in humans. Nat Genet 1998; 20: 278-280. 